\title{
Perinatal SSRI medications and offspring hippocampal plasticity: interaction with maternal stress and sex
}

\author{
Jodi L. Pawluski ${ }^{1} \cdot$ Mary Gemmel $^{2}$
}

Published online: 6 April 2018

(C) Hellenic Endocrine Society 2018

\begin{abstract}
There is growing use of selective serotonin reuptake inhibitor antidepressant (SSRI) medications during the perinatal period to treat maternal affective disorders. Perinatal SSRI exposure can have a long-term impact on offspring neuroplasticity and behavioral development that remains to be fully elucidated. This mini-review will summarize what is known about the effects of perinatal SSRIs on plasticity in the developing hippocampus, taking into account the role that maternal stress and depression may have. Emerging clinical findings and research in animal models will be discussed. In addition, sexually differentiated effects will be highlighted, as recent work shows that male offspring are often more sensitive to the effects of maternal stress, whereas female offspring can be more sensitive to perinatal SSRIs. Potential mechanisms behind these changes and aims for future research will also be discussed. Understanding the impact of perinatal SSRIs on neuroplasticity will provide better insight into the long-term effects of such medications on the health and well-being of both mother and child and may improve therapeutic approaches for maternal mood disorders during the perinatal period.
\end{abstract}

Keywords Antidepressants $\cdot$ Perinatal depression $\cdot$ Prenatal stress $\cdot$ Neurogenesis $\cdot$ Hippocampus $\cdot$ Adolescence $\cdot$ Sex differences $\cdot 5$-HT $\cdot$ Serotonin

\section{Introduction}

Up to $20 \%$ of women are diagnosed with depression or anxiety during the perinatal period [1-4]. These affective disorders can have detrimental effects for both the mother and developing child, thus treatment is needed $[4,5]$. Many antidepressant treatments are available to treat maternal affective disorders, with selective serotonin reuptake inhibitor medications (SSRIs) being the recommended first line of treatment $[4,6$, 7]. SSRIs, with the exception of paroxetine, are considered safe for use during pregnancy as they have no major teratogenic

This work was presented at the 1st Conference of the Institute of Stress Biology and Medicine (ISBM) entitled: "Systems Biology-Medicine and Stress", held in Athens on 20-22 January, 2017.

Jodi L. Pawluski

Jodi-lynn.pawluski@univ-rennes1.fr; j.pawluski@gmail.com

1 Univ Rennes, Inserm, EHESP, Irset (Institut de Recherche en Santé, Environnement et Travail), UMR_S 1085, F-35000 Rennes, France

2 Department of Biological Sciences, Ohio University, Athens, OH, USA effects on the fetus [8]. SSRI prescription rates for pregnant women in industrialized countries (including Canada, Iceland, Denmark, Sweden, UK, Italy, the Netherlands, and France) range between 2 and $7 \%$ and between 5 and $13 \%$ in Australia and the USA [6, 7, 9-12]. These medications are expected to promote maternal mental health and thus promote the health and well-being of both the mother and child [13]. However, SSRIs can cross the placenta and, to a lesser degree, are found in breast milk, thereby modulating the serotonergic environment for the developing fetus and having potentially long-term effects on neurodevelopment [14-17].

Clinical research has shown that children exposed to SSRIs prenatally may be at increased risk of behavioral abnormalities including attentional deficits, neuropsychiatric disorders, and neurodevelopmental disorders [18-20]. As the hippocampus is a key mediator of behavior and cognition and receives substantial serotoninergic innervation, such effects are likely linked to SSRI-related changes in hippocampal development. Therefore, this mini-review will focus on how perinatal SSRIs affect plasticity in the developing hippocampus. First, we will briefly review how SSRIs during the perinatal period may alter the developing serotoninergic system, and then, we will review what is known about perinatal SSRI effects on 
biomarkers of neuroplasticity in clinical studies and measures of hippocampus plasticity from work in laboratory rodent models. Early-life exposure to maternal mental illness, in the presence of SSRIs, can also have a persistent effect on offspring neurobehavioral outcomes $[21,22]$. Thus, the implications of both perinatal SSRIs and maternal stress-related disorders will be discussed in terms of offspring outcomes where possible $[23,24]$. Understanding the long-term effect of perinatal SSRIs on hippocampus plasticity will aid in understanding the risks and benefits of exposure to these medications during the perinatal period.

\section{Serotonin, SSRIs, and the developing hippocampus}

During development, serotonin (5-hydroxytryptamine or 5HT) plays an important role in regulating proliferation, migration, and differentiation of neurons as well as axonal connectivity and synaptic pruning [25-27]. A number of studies indicate that serotonin signaling during early life is critically involved in the development of neuronal circuits [27-32]. Hence, any changes to serotonin levels and/or serotonergic system functioning during the perinatal period could disrupt the development of not only the serotonergic system in infants but also countless brain regions which receive serotonergic input, such as the hippocampus.

SSRIs act by blocking the serotonin transporter (SERT) on the presynaptic neuronal membrane and subsequently reducing serotonin reuptake, increasing serotonin's concentration in the synaptic cleft and aiding in chemical transmission between neurons which can have mood-stabilizing effects. As perinatal SSRIs target maternal SERT activity and are present in both the placenta and, at much lower levels, in breast milk [33], the use of such medications during pregnancy and lactation suggests an effect on infant neural development. The consequences of perinatal SSRI exposure may not occur immediately after birth but may manifest later during childhood, adolescence, or even in adulthood [14, 34, 35].

Perinatal SSRI treatment can increase hippocampal serotonin levels in adolescent and adult male mice [36] and preadolescent male and female offspring [37]. Interestingly, earlylife exposure to SSRIs also normalizes hippocampal levels of serotonin and serotonin's metabolite 5-hydroxyindolacetic acid (5-HIAA) in prenatally restraint stressed offspring at weaning [38], serotonergic transmission in the ventral hippocampus of adolescent rat offspring after prenatal dexamethasone treatment [39], and serotonin turnover (via 5-HIAA/5HT ratios) in the hippocampus of prenatally stressed male mouse offspring (Table 1) [36]. Although not well studied in the hippocampus, perinatal SSRIs do affect serotonergic receptors in cortical and hypothalamic brain regions, and thus, many layers of the serotonergic system are likely altered by perinatal SSRIs [49-51]. It should not be forgotten that maternal stress can also have enduring effects on serotonin, even when combined with perinatal SSRIs. Recently, we have shown that pregestational chronic unpredictable stress results in significantly reduced hippocampal serotonin levels in preadolescent offspring, particularly in female offspring, regardless of perinatal fluoxetine exposure [17, 34]. Others have shown that pregestational stress increases serotonin levels and decreases serotonin transporter expression in the hippocampus of fetal rats [52] and that prenatal stress increases serotonin in weanling offspring, regardless of fluoxetine exposure. Changes in early-life serotonin, and serotonin transporters, may have enduring effects on serotonergic functioning of offspring later in life. Together, these findings show that there are long-term effects of both SSRIs and maternal stress on the serotonin system of the hippocampus and that the timing and duration of maternal stress (pregestational versus prenatal), as well as offspring age and sex, may mediate these effects on the developing serotonergic system. Therefore, understanding how fluctuations in serotonin and the serotonergic system impact developing neuroplasticity via early-life SSRIs, and maternal stress, is critical in integrating what we know about hippocampal plasticity in offspring.

\section{Perinatal SSRIs and biomarkers of neuroplasticity in clinical research}

Findings from clinical studies are starting to point to an effect of prenatal SSRIs on fetal and neonatal neurodevelopment by investigating biomarkers in peripheral fluids related to central nervous system development. It has been reported that prenatal SSRIs decrease S100B, an astroglial-specific Ca2+-binding protein $[53,54]$ in human neonates at birth [55]. S100B mediates the positive outgrowth and survival of neurons $[56,57]$ and stimulates glial cell proliferation [58]; therefore, S100B levels in human biological fluids may be a useful indicator of brain maturation and the impact of prenatal drug exposure on neural development [55]. Since this reduction in S100B occurs when controlling for maternal levels of depression, which serves as a confounding variable in clinical work [5], such outcomes imply a significant effect of perinatal SSRI exposure itself on potential markers of neurodevelopment. In addition, reelin levels are decreased in neonates prenatally exposed to SSRIs [59]. Reelin is an important glycoprotein which plays a critical role in neuronal migration and positioning during neurodevelopment, and these findings further suggest an effect of early-life changes in serotonin, via SSRIs, on the brain of developing offspring. Recent imaging data has confirmed that prenatal SSRIs do indeed alter the developing brain, at least in very preterm infants, with prenatal SSRI-exposed infants showing decreased activity in the basal ganglia and thalamus [60]. However, due to limitations in the ability of clinical 


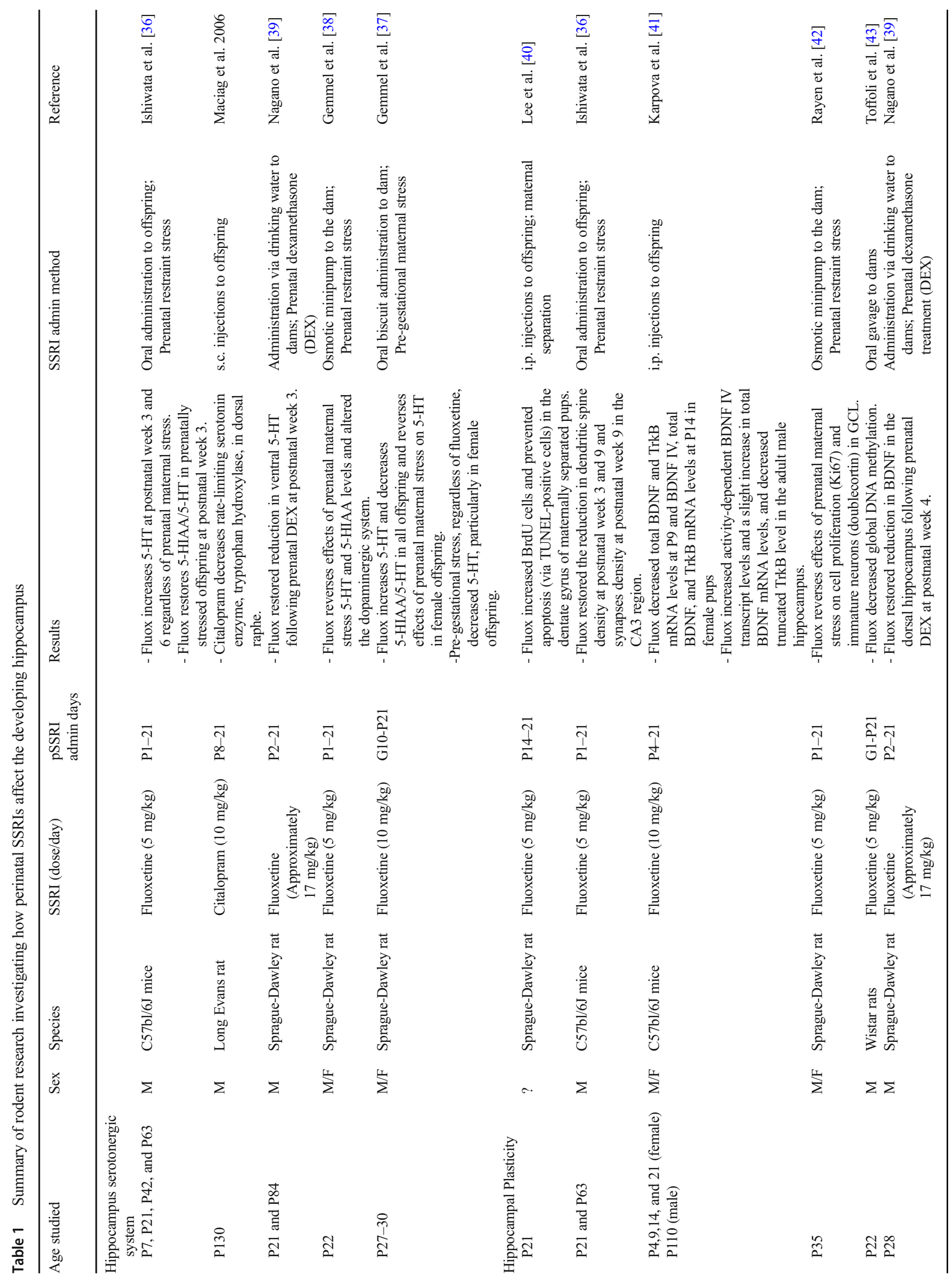




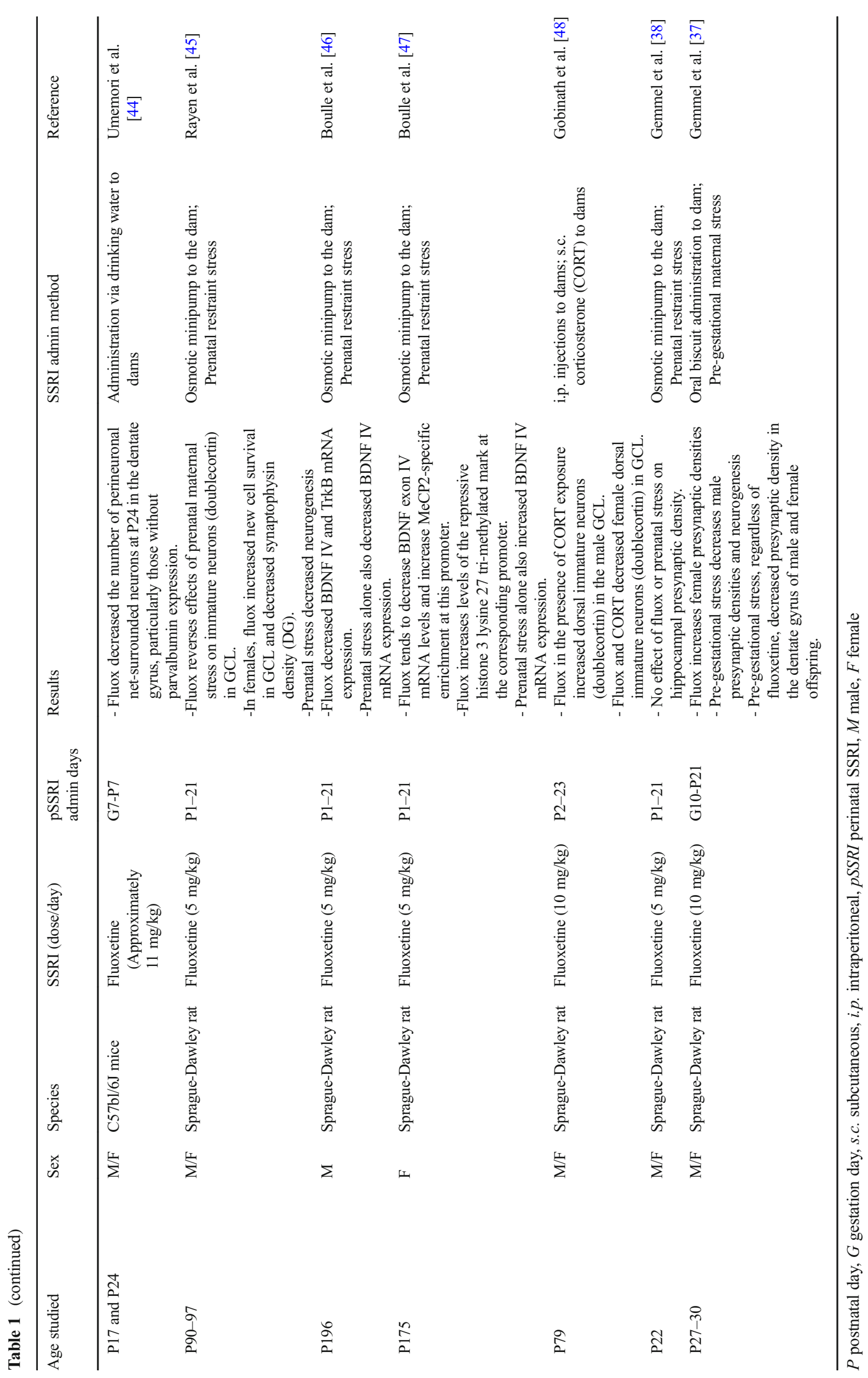


research to investigate central neurodevelopment in SSRIexposed offspring, animal models have been used to gain insight into the specific effects of perinatal SSRIs on neurogenesis and synaptic plasticity in the hippocampus. Below is a summary of findings on how perinatal SSRIs affect the developing hippocampus of male and female offspring.

\section{Perinatal SSRIs and plasticity in the hippocampus}

With clinical work pointing to an effect of perinatal SSRIs on both S100B and reelin, key players in neural migration and plasticity, it is not surprising that there is growing interest in the long-term impact of SSRIs on hippocampal neurogenesis and plasticity. The hippocampus is one of two brain regions where there is a remarkably high rate of neurogenesis throughout the lifespan (the other region being the subventricular zone) $[61,62]$. These new neurons, and their ability to form and establish new connections in the hippocampus, play an important role in learning and memory, stress regulation, and, as determined more recently, social behaviors [62-66]. The hippocampus consists of three main regions: cornu ammonis 1 (CA1), cornu ammonis 3 (CA3), and the dentate gyrus, the latter being a site with a high rate of continuous neurogenesis in adulthood (Fig. 1) [67, 68]. Understanding how neurogenesis and neuroplasticity may shift following perinatal SSRIs will further clarify the role that early-life exposures have on neurobehavioral development.

To date, findings have highlighted the long-term effects of perinatal SSRIs on hippocampal plasticity in rodent offspring, particularly after maternal stress. These studies have primarily focused on measures of brain-derived neurotrophic factor (BDNF), neurogenesis, and synaptic modifications in the hippocampus. Of particular importance are alterations of BDNF signaling, epigenetic changes in the BDNF gene, and changes in hippocampal plasticity, which have been strongly implicated in the pathophysiology and treatment of mood disorders during adulthood [69, 70]. Developmental exposure to SSRIs has resulted in long-term impact on BDNF in offspring: early developmental treatment with fluoxetine can induce longlasting behavioral impairment, via increased inhibition to stressful events and reduced behavioral despair, accompanied with upregulation of hippocampal BDNF mRNA and TrkB mRNA levels in adult male mice [41] and a decrease in global DNA methylation in the hippocampus of male rats [43]. Serotonin transporter (5-HTT) knockout rats, which have elevated levels of serotonin throughout life, display decreased BDNF levels in the hippocampus, concomitant with increased DNA methylation at BDNF promoters IV and VI [71]. An exact comparison between perinatal SSRI exposure and 5HTT KO rat models with regard to BDNF levels and methylation status has not been carried out; however, it is likely that the apparent differences between models on BDNF effects in the hippocampus are due to the fact that 5-HTT KO animals have elevated levels of serotonin throughout life and not just during the perinatal period. While this work suggests an effect of perinatal SSRI exposure on BDNF, these three previous studies did not investigate female offspring. We have recently reported in adult female rat offspring that early-life exposure to SSRIs (fluoxetine) increases immobility in the forced swim test, decreases hippocampal BDNF exon IV mRNA levels, and increases levels of the repressive histone 3 lysine 27 tri-methylated mark at the corresponding promoter [46]. We also found a significant negative correlation between hippocampal BDNF exon IV mRNA levels and immobility in the forced swim test, with higher hippocampal BDNF mRNA expression being associated with less immobility in this test [46]. In adult male offspring, developmental fluoxetine exposure decreased BDNF IV and TrkB mRNA expression in the hippocampus and these changes were not associated with changes in the immobility measure [47]. In addition, adult male offspring showed an enduring effect of prenatal stress in decreasing hippocampal BDNF IV mRNA expression [47], further demonstrating that these effects are sexually differentiated following SSRI exposure. Regardless, perinatal SSRI effects on epigenetic modifications and changes to BDNF signaling, a critical growth factor required for neuronal growth and development, suggest an impact on additional aspects of neurodevelopment such as neurogenesis and neuroplasticity.

Early-life exposure to SSRIs reduces cell proliferation in the hippocampus of adolescent offspring [42]. However, when combined with a model of maternal stress, early-life exposure to SSRIs prevents the effects of maternal stress on
Fig. 1 Photomicrographs of $\mathbf{A}$ ) synaptophysin-immunoreactivity (ir) in the hippocampus $(2 \times)$ and B) immature neurons in the granule call layer (doublecortinir) $(40 \times)$ of preadolescent rat offspring. $\mathrm{CA}=$ cornu ammonis, $\mathrm{DG}=$ dentate gyrus. The arrows indicate immature neurons. Scale bar $=200 \mu \mathrm{m}(\mathbf{a})$ and $10 \mu \mathrm{m}(\mathbf{b})$
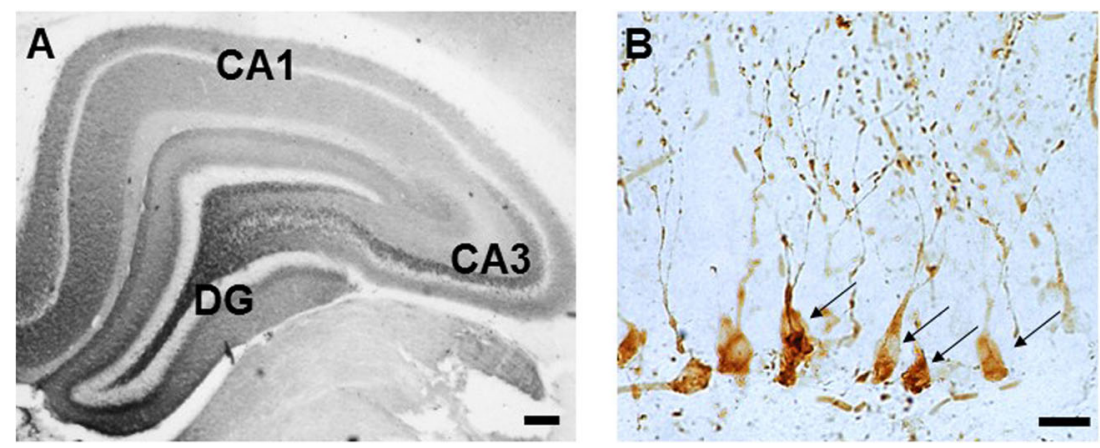
hippocampal neurogenesis (immature neurons) in adolescence and adulthood $[42,45]$. These effects occur in both male and female offspring. Others have shown similar effects with postnatal SSRIs preventing the reduction in hippocampal cell proliferation and increased cell death observed in juvenile rat offspring subjected to premature maternal separation [40]. Developmental exposure to fluoxetine can also have a sexually differentiated effect on the number of immature neurons after maternal corticosterone treatment [48], with maternal postpartum fluoxetine increasing the density of immature neurons via doublecortin expression in the hippocampus of adult male offspring but decreasing the density of immature neurons in adult female offspring. Interestingly, additional reports found no effects of perinatal fluoxetine on hippocampal neurogenesis in the dorsal hippocampus at weaning or in preadolescent offspring [37, 38]. Thus, it appears that after adolescence and puberty, the effects of perinatal SSRIs on hippocampal neurogenesis and plasticity become pronounced and sexually differentiated.

With regard to perinatal SSRI effects on spine densities and synaptic proteins, these effects appear to be primarily dependent on offspring age and sex. For example, early postnatal treatment with fluoxetine reverses the reduction in CA3 spine and synapse density observed in prenatally stressed juvenile and adolescent male mice (females were not studied) [36]. However, in rat offspring at weaning, we found no effect of early-life exposure to fluxotine on presynaptic protein densities (via synaptophysin-immunoreactivity) in the CA3 or dentate gyrus [38]. Effects on spine density appear to emerge during preadolescence, with preadolescent female, not male, rat offspring perinatally exposed to fluoxetine showing increased hippocampal presynaptic density in the dentate gyrus [37]. These effects are region-specific, with perinatal fluoxetine exposure increasing CA2 density in preadolescent females but decreasing such density in combination with pregestational maternal stress exposure. As previously mentioned, although females seem particularly sensitive to the effects of perinatal fluoxetine exposure, male offspring appear particularly sensitive to maternal stress and depression [37]. For example, preadolescent males, but not females, exposed to perinatal fluoxetine show reductions in presynaptic density and immature neurons in the dentate gyrus following pregestational maternal stress [37]. Furthermore, SSRI exposure appears to affect the developmental trajectory of synaptic protein density, with adult female, but not male, offspring exposed during lactation/suckling to SSRIs showing significantly reduced presynaptic density in the dentate gyrus [45]. This enduring effect of SSRIs on synaptic proteins extends recent work showing that perinatal fluoxetine exposure can reduce perineuronal nets in the CA1 and DG region of juvenile mice hippocampi [44]. In addition, postnatal fluoxetine treatment significantly alters gene expression related to hippocampal synaptic functioning and neurogenesis in selectively bred "Low Responder" rats predisposed for increased anxiety and behavioral abnormalities [72]. Thus, there is a likely role for perinatal fluoxetine exposure in altering hippocampal synaptic plasticity during development: effects that are likely sexually differentiated and age-dependent (Table 1).

\section{SSRIs and sex effects on hippocampal plasticity}

As mentioned above, there is a persistent effect of sex in mediating perinatal SSRI exposure effects on hippocampal plasticity in adulthood. For example, adult female offspring, but not male offspring, exposed early in life to SSRIs have increased new cell survival, decreased BDNF mRNA expression, and reductions in hippocampal synaptophysin density in the granule cell layer [45-47]. Others have shown that maternal postnatal fluoxetine treatment increases adult male immature neuron density in the hippocampus via doublecortin expression while reducing such density in the adult female hippocampus [48]. Changes in steroid hormone levels during puberty likely contribute to sex-dependent SSRI sensitivity and structural changes in the hippocampus as a result of altered estrogen and androgen receptor expression [73-75]. Serotonin plays a key role in sexual differentiation through its role in the development of the hypothalamic-pituitary-gonadal (HPG) axis $[76,77]$. The inhibition of the natural drop in serotonin in the first week of life resulting from SSRI exposure likely antagonizes the perinatal masculinization effects of testosterone during the second and/or third week postpartum and alters feminization of the brain. Previous work has shown that postnatal stimulation of serotonin synthesis, by injection of L-tryptophan, inhibits female sexual behavior and has an inhibitory effect on postnatal "organization" of female sexual behavior as well as on "activation" of female sexual behavior in adulthood [76, 77]. In addition, a defeminization of sexually dimorphic brain structures in females results from early-life stimulation of the serotonin synthesis [78] and, conversely, treatment with parachlorophenylalanine, a serotonin synthesis inhibitor, enhances masculinization and defeminization [78, 79]. Therefore, sexually differentiated effects of perinatal SSRIs on hippocampal plasticity may be a result of changes to the serotonergic system early in life, as serotonin plays a role in sexual differentiation of the brain via development of the HPG axis $[76,77]$.

\section{The role of maternal stress}

As shown above, the effects of perinatal SSRIs can differ in the presence of maternal stress in some regards, the effects of maternal stress being more pronounced and enduring than the effects of SSRIs. For example, maternal stress, regardless of 
perinatal SSRI exposure, significantly reduces presynaptic densities in preadolescent male, but not female, offspring and decreases the number of immature neurons in the granule cell layer of dentate gyrus in preadolescent and adult male, but not female, offspring [45]. Interestingly, pregestational maternal stress effects on offspring are in agreement with a number of studies showing that perinatal maternal stressors reduce hippocampal neurogenesis and plasticity later in life, particularly in males $[80,81]$. In line with this, an increasing amount of clinical research is reporting that child development outcomes are often more affected by maternal depression and associated risk factors prior to conception than by perinatal SSRI exposure [5, 21, 22]. Thus, a mother's level of stress and depression during the perinatal period and prior to gestation can have a long-term impact on neurobehavioral outcomes in offspring, regardless of, or in addition to, perinatal SSRI effects. More research is needed to investigate additional therapeutic approaches which treat the effects of perinatal depression in both mothers and offspring.

\section{Summary and future directions}

It is clear that perinatal SSRIs can have a long-term effect on plasticity in the hippocampus of offspring. Perinatal SSRIs can at times protect against the effect of maternal stress, have effects independent of maternal stress, and have effects on offspring that are sexually differentiated. The effects of perinatal SSRIs on hippocampal plasticity may be both direct, due to perinatal drug exposure, and indirect, with effects mediated by maternal care as well as SSRI effects on the placenta [17, 82-84]. Apart from effects on hippocampal plasticity, a growing body of literature shows that perinatal exposure to SSRIs affects a number of neuroendocrine systems and neurobehavioral outcomes which may be linked to the hippocampus. For example, developmental exposure to SSRIs alters the HPG axis $[79,85]$ and the hypothalamic-pituitary-adrenal axis $[36,86,87]$ and has been linked to poor social behaviors and increased mood disorders in children [34]. However, there are also persistent effects of maternal stress and maternal mood on childhood outcomes, even with maternal treatment of SSRIs [5, 21, 22].

What is abundantly clear is that untreated maternal depression is not an option for the mother or developing child. SSRIs are often the first-line treatment for maternal mood disorders and these medications are more effective if combined with psychotherapy. However, there are a number of treatments available that may also be beneficial to the mother, such as psychotherapy alone, parenting classes, exercise, and diet change $[13,88,89]$. Regardless, the goal is to effectively treat the mother as the risk of untreated maternal depression outweighs the risk of the SSRI treatment exposure. On the other hand, many pregnant women prescribed SSRIs also remain depressed and anxious and this is detrimental to the mother, child, and family. Therefore, SSRI treatment for maternal affective disorders during pregnancy should be treated on an individualized basis which takes into account depression severity, likelihood of treatment response and probability of adverse fetal effects, and individual patient values and health [90]. The data reviewed here suggest that future treatment for maternal affective disorders may also need to consider offspring sex as well as SSRI timing and dosage.

Unfortunately, to date, there has been very little research on the neurobiology of maternal mental illness, even though one in seven women suffer from perinatal depression or clinical levels of perinatal anxiety [4]. Thus, more research is needed to understand the mechanisms behind maternal mental illness in order to develop effective and safe treatments for these disorders. Only then will we be able to improve the health and well-being of the mother and child.

Acknowledgements JLP is funded by a Brain and Behavior Foundation NARSAD Young Investigator Grant and SAD funding from the Region of Bretagne.

Conflicts of Interest The authors have no conflicts of interests to report.

Ethical Considerations As this is a minireview of the literature, ethical approval was not required.

\section{References}

1. Almond P (2009) Postnatal depression: a global public health perspective. Perspect Public Health 129:221-227

2. Burke HM, Davis MC, Otte C, Mohr DC (2005) Depression and cortisol responses to psychological stress: a meta-analysis. Psychoneuroendocrinology 30:846-856

3. Leung BM, Kaplan BJ (2009) Perinatal depression: prevalence, risks, and the nutrition link-a review of the literature. J Am Diet Assoc 109:1566-1575

4. Pawluski J, Lonstein JS, Fleming AS (2017) The neurobiology of postpartum anxiety and depression. Trends Neurosci 40:106-120

5. Oberlander TF, Zwaigenbaum L (2017) Disentangling maternal depression and antidepressant use during pregnancy as risks for autism in children. JAMA 317:1533-1534

6. Oberlander TF, Warburton W, Misri S, Aghajanian J, Hertzman C (2006) Neonatal outcomes after prenatal exposure to selective serotonin reuptake inhibitor antidepressants and maternal depression using population-based linked health data. Arch GenPsychiatry 63: 898-906

7. Cooper WO, Willy ME, Pont SJ, Ray WA (2007) Increasing use of antidepressants in pregnancy. Am J Obstet Gynecol 196:544 e1-5

8. Gentile $S$ (2005) The safety of newer antidepressants in pregnancy and breastfeeding. Drug Saf 28:137-152

9. Hayes RM, Wu P, Shelton RC (2012) Maternal antidepressant use and adverse outcomes: a cohort study of 228,876 pregnancies. Am J Obstet Gynecol 207:49 e41-49

10. Charlton RA, Jordan S, Pierini A (2015) Selective serotonin reuptake inhibitor prescribing before, during and after pregnancy: a population-based study in six European regions. BJOG 122: $1010-1020$ 
11. Zoega H, Kieler H, Nørgaard M (2015) Use of SSRI and SNRI antidepressants during pregnancy: a population-based study from Denmark, Iceland, Norway and Sweden. PLoS One 10:e0144474

12. Lupattelli A, Spigset O, Twigg MJ (2014) Medication use in pregnancy: a cross-sectional, multinational web-based study. BMJ Open 4:e04365

13. Kim DR, Epperson CN, Weiss AR, Wisner KL (2014) Pharmacotherapy of postpartum depression: an update. Expert Opin Pharmacother 15:1223-1234

14. Oberlander TF, Gingrich JA, Ansorge MS (2009) Sustained neurobehavioral effects of exposure to SSRI antidepressants during development: molecular to clinical evidence. Clin Pharmacol Ther 86: 672-677

15. Homberg JR, Schubert D, Gaspar P (2010) New perspectives on the neurodevelopmental effects of SSRIs. Trends Pharmacol Sci 31: $60-65$

16. Glover ME, Clinton SM (2016) Of rodents and humans: a comparative review of the neurobehavioral effects of early life SSRI exposure in preclinical and clinical research. Int J Dev Neurosci 51:50 72

17. Gemmel M, Hazlett M, Bögi E (2017) Perinatal fluoxetine effects on social play, the HPA system, and hippocampal plasticity in preadolescent male and female rats: interactions with pre-gestational maternal stress. Psychoneuroendocrinology 84:159-171

18. Clements CC, Castro VM, Blumenthal SR (2015) Prenatal antidepressant exposure is associated with risk for attention-deficit hyperactivity disorder but not autism spectrum disorder in a large health system. Mol Psychiatry 20:727-734

19. Man KK, Tong HH, Wong LY, Chan EW, Simonoff E, Wong IC (2015) Exposure to selective serotonin reuptake inhibitors during pregnancy and risk of autism spectrum disorder in children: a systematic review and meta-analysis of observational studies. Neurosci Biobehav Rev 49:82-89

20. Oberlander TF, Reebye P, Misri S, Papsdorf M, Kim J, Grunau RE (2007) Externalizing and attentional behaviors in children of depressed mothers treated with a selective serotonin reuptake inhibitor antidepressant during pregnancy. Arch Pediatr Adolesc Med 161: 22-29

21. Brown HK, Reebye P, Misri S, Papasdorf M, Kim J, Gruanau RE (2017) Association between serotonergic antidepressant use during pregnancy and autism Spectrum disorder in children. JAMA 317: 1544-1552

22. Mezzacappa A, Lasica PA, Gianfagna F (2017) Risk for autism Spectrum disorders according to period of prenatal antidepressant exposure: a systematic review and meta-analysis. JAMA Pediatr 171:555-563

23. Haim A, Sherer M, Leuner B (2014) Gestational stress induces persistent depressive-like behavior and structural modifications within the postpartum nucleus accumbens. Eur J Neurosci 40: 3766-3773

24. O'Mahony SM, Myint AM, van den Hove D, Desbonnet L, Steinbusch H, Leonard BE (2006) Gestational stress leads to depressive-like behavioural and immunological changes in the rat. Neuroimmunomodulation 13:82-88

25. Gaspar P, Cases O, Martoreaux L (2003) The developmental role of serotonin: news from mouse molecular genetics. Nat Rev Neurosci 4:1002-1012

26. Herlenius E, Lagercrantz H (2004) Development of neurotransmitter systems during critical periods. Exp Neurol 190(Suppl 1):8-21

27. Whitaker-Azmitia PM (2001) Serotonin and brain development: role in human developmental diseases. Brain Res Bull 56:479-485

28. Ansorge MS, Morelli E, Gingrich JA (2008) Inhibition of serotonin but not norepinephrine transport during development produces delayed, persistent perturbations of emotional behaviors in mice. $\mathrm{J}$ Neurosci 28:199-207
29. Ansorge MS, Zhou M, Lira A, Hen R, Gingrich JA (2004) Earlylife blockade of the 5-HT transporter alters emotional behavior in adult mice. Science 306:879-881

30. Fernandez SP, Muzerelle A, Scotto-Lomassese S (2016) Constitutive and acquired serotonin deficiency alters memory and hippocampal synaptic plasticity. Neuropsychopharmacology 42: $512-523$

31. Kurzepa S, Bojanek J (1965) The 5HT level and MAO activity in rat brain during development. Biol Neonat 8:216-221

32. Baker PC, Quay WB (1969) 5-hydroxytryptamine metabolism in early embryogenesis, and the development of brain and retinal tissues. A review. Brain Res 12:273-295

33. Kristensen JH, Ilett KF, Hackett LP, Yapp P, peach M, Begg EJ (1999) Distribution and excretion of fluoxetine and norfluoxetine in human milk. Br J Clin Pharmacol 48:521-527

34. Gemmel M, Bögi E, Ragan C (2017) Perinatal selective serotonin reuptake inhibitor medication (SSRI) effects on social behaviors, neurodevelopment and the epigenome. Neurosc Biobehav Rev 85: 102-116

35. Pawluski JL (2012) Perinatal selective serotonin reuptake inhibitor exposure: impact on brain development and neural plasticity. Neuroendocrinology 95:39-46

36. Ishiwata H, Shiga T, Okado N (2005) Selective serotonin reuptake inhibitor treatment of early postnatal mice reverses their prenatal stress-induced brain dysfunction. Neuroscience 133:893-901

37. Gemmel HM, Bögi E (2017) Under review perinatal fluoxetine effects on social play, the HPA system, and hippocampal plasticity in pre-adolescent male and female rats: interactions with maternal stress. Psychoneuroendocrinology 84:159-171

38. Gemmel M, Rayen I, Lotus T (2016) Developmental fluoxetine and prenatal stress effects on serotonin, dopamine, and synaptophysin density in the PFC and hippocampus of offspring at weaning. Dev Psychobiol 58:315-327

39. Nagano M, Liu M, Inagaki H, Kawada T, Suzuki H (2012) Early intervention with fluoxetine reverses abnormalities in the serotonergic system and behavior of rats exposed prenatally to dexamethasone. Neuropharmacology 63:292-300

40. Lee HJ, Kim JW, Yim SV (2001) Fluoxetine enhances cell proliferation and prevents apoptosis in dentate gyrus of maternally separated rats. Mol Psychiatry 6(610):725-728

41. Karpova NN, Lindholm J, Pruunsild P, Timmusk T, Castrén E (2009) Long-lasting behavioural and molecular alterations induced by early postnatal fluoxetine exposure are restored by chronic fluoxetine treatment in adult mice. Eur Neuropsychopharmacol 19: 97-108

42. Rayen I, van den Hove DL, Prickaerts J, Steinbusch HW, Pawluski JL (2011) Fluoxetine during development reverses the effects of prenatal stress on depressive-like behavior and hippocampal neurogenesis in adolescence. PLoS One 6:e24003

43. Toffoli LV, Rodriguew GM Jr, Oliveira JF (2014) Maternal exposure to fluoxetine during gestation and lactation affects the DNA methylation programming of rat's offspring: modulation by folic acid supplementation. Behav Brain Res 265:142-147

44. Umemori J, Winkel F, Castrén E, Karpova NN (2015) Distinct effects of perinatal exposure to fluoxetine or methylmercury on parvalbumin and perineuronal nets, the markers of critical periods in brain development. Int J Dev Neurosci 44:55-64

45. Rayen I, Gemmel M, Pauley G, Steinbusch HW, Pasluski JL (2015) Developmental exposure to SSRIs, in addition to maternal stress, has long-term sex-dependent effects on hippocampal plasticity. Psychopharmacology 232:1231-1244

46. Boulle F, Pawluski JL, Homberg JR (2016) Developmental fluoxetine exposure increases behavioral despair and alters epigenetic regulation of the hippocampal BDNF gene in adult female offspring. Horm Behav 80:47-57 
47. Boulle F, Pawluski JL, Homberg JR (2016) Prenatal stress and early-life exposure to fluoxetine have enduring effects on anxiety and hippocampal BDNF gene expression in adult male offspring. Dev Psychobiol 58:427-438

48. Gobinath AR, Workman JL, Chow C, Lieblich SE, Galea LA (2016) Maternal postpartum corticosterone and fluoxetine differentially affect adult male and female offspring on anxiety-like behavior, stress reactivity, and hippocampal neurogenesis. Neuropharmacology 101:165-178

49. Olivier JD, Akerud H, Kaihola H (2013) The effects of maternal depression and maternal selective serotonin reuptake inhibitor exposure on offspring. Front Cell Neurosci 7:73

50. Cabrera TM, Battaglia G (1994) Delayed decreases in brain 5hydroxytryptamine $2 \mathrm{~A} / 2 \mathrm{C}$ receptor density and function in male rat progeny following prenatal fluoxetine. J Pharmacol Exp Ther 269:637-645

51. Sarkar A, chachra P, Vaidya VA (2014) Postnatal fluoxetine-evoked anxiety is prevented by concomitant $5-\mathrm{HT} 2 \mathrm{~A} / \mathrm{C}$ receptor blockade and mimicked by postnatal $5-\mathrm{HT} 2 \mathrm{~A} / \mathrm{C}$ receptor stimulation. Biol Psychiatry 76:858-868

52. Huang Y, Xu H, Li H, Yang H, Chen Y, Shi X (2012) Pregestational stress reduces the ratio of 5-HIAA to 5-HT and the expression of 5-HT1A receptor and serotonin transporter in the brain of foetal rat. BMC Neurosci 13:22

53. Haring JH, Hagan A, Olson J, Rodgers B (1993) Hippocampal serotonin levels influence the expression of S100 beta detected by immunocytochemistry. Brain Res 631:119-123

54. Whitaker-Azmitia PM, Murphy R, Azmitia EC (1990) Stimulation of astroglial 5-HT1A receptors releases the serotonergic growth factor, protein S-100, and alters astroglial morphology. Brain Res 528:155-158

55. Pawluski JL, Galea LA, Brain U, Papsdorf M, Oberlander TF (2009) Neonatal S100B protein levels after prenatal exposure to selective serotonin reuptake inhibitors. Pediatrics 124:e662-e670

56. Bhattacharyya A, Oppenheim RW, Prevette D, Moore BW, Brackenbury R, Ratner N (1992) S100 is present in developing chicken neurons and Schwann cells and promotes motor neuron survival in vivo. J Neurobiol 23:451-466

57. Gonzalez-Martinez T, Perez-Piñera P, Díaz-Esnal B, Vega JA (2003) S-100 proteins in the human peripheral nervous system. Microsc Res Tech 60:633-638

58. Selinfreund RH, Barger SW, Pledfer WJ, van Eldik LJ (1991) Neurotrophic protein S100 beta stimulates glial cell proliferation. Proc Natl Acad Sci U S A 88:3554-3558

59. Brummelte S, Galea LA, Devlin AM, Oberlander TF (2013) Antidepressant use during pregnancy and serotonin transporter genotype (SLC6A4) affect newborn serum reelin levels. Dev Psychobiol 55:518-529

60. Podrebarac SK, Duerden EG, Chau V (2017) Antenatal exposure to antidepressants is associated with altered brain development in very preterm-born neonates. Neuroscience 342:252-262

61. Eriksson PS, Perfilieva E, Björk-Eriksson T (1998) Neurogenesis in the adult human hippocampus. Nat Med 4:1313-1317

62. Pawluski JL, Brummelte S, Barha CK, Crozier TM, Galea LA (2009) Effects of steroid hormones on neurogenesis in the hippocampus of the adult female rodent during the estrous cycle, pregnancy, lactation and aging. Front Neuroendocrinol 30:343-357

63. Hammels C, Prickaerts J, Kenis G (2015) Differential susceptibility to chronic social defeat stress relates to the number of Dnmt3aimmunoreactive neurons in the hippocampal dentate gyrus. Psychoneuroendocrinology 51:547-556

64. Dalla C, Whetstone AS, Hodes GE, Shors TJ (2009) Stressful experience has opposite effects on dendritic spines in the hippocampus of cycling versus masculinized females. Neurosci Lett 449:5256
65. Leuner B, Gould E (2010) Structural plasticity and hippocampal function. Annu Rev Psychol 61:111-140

66. Djavadian RL (2004) Serotonin and neurogenesis in the hippocampal dentate gyrus of adult mammals. Acta Neurobiol Exp (Wars) 64: 189-200

67. Altman J (1962) Are new neurons formed in the brains of adult mammals? Science 135:1127-1128

68. Altman J, Das GD (1967) Postnatal neurogenesis in the Guinea-pig. Nature 214:1098-1101

69. Boulle F, van den Hove DL, Jakob SB (2012) Epigenetic regulation of the BDNF gene: implications for psychiatric disorders. Mol Psychiatry 17:584-596

70. Castren E, Rantamäki T (2010) The role of BDNF and its receptors in depression and antidepressant drug action: reactivation of developmental plasticity. Dev Neurobiol 70:289-297

71. Molteni R, Cattaneo A, Calabrese F (2010) Reduced function of the serotonin transporter is associated with decreased expression of BDNF in rodents as well as in humans. Neurobiol Dis 37:747-755

72. Glover ME, Pugh PC, Jackson NL (2015) Early-life exposure to the SSRI paroxetine exacerbates depression-like behavior in anxiety/ depression-prone rats. Neuroscience 284:775-797

73. Galea LA, Spritzer MD, Barker JM, Pawluski JL (2006) Gonadal hormone modulation of hippocampal neurogenesis in the adult. Hippocampus 16:225-232

74. Tabori NE, Stewart LS, Znamensky V (2005) Ultrastructural evidence that androgen receptors are located at extranuclear sites in the rat hippocampal formation. Neuroscience 130:151-163

75. Weiland NG, Orikasa C, Hayashi S, McEwen BS (1997) Distribution and hormone regulation of estrogen receptor immunoreactive cells in the hippocampus of male and female rats. J Comp Neurol 388:603-612

76. Döhler KD, Jarzab B, Sckmöller PM (1991) Influence of neurotransmitters on sexual differentiation of brain structure and function. Exp Clin Endocrinol 98:99-109

77. Jarzab B, Dohler KD (1984) Serotoninergic influences on sexual differentiation of the rat brain. Prog Brain Res 61:119-126

78. Wilson CA, Pearson JR, Hunter AJ, Tuohy PA, Payne AP (1986) The effect of neonatal manipulation of hypothalamic serotonin levels on sexual activity in the adult rat. Pharmacol Biochem Behav 24:1175-1183

79. Rayen I, Steinbusch HW, Charlier TD, Pawluski JL (2013) Developmental fluoxetine exposure and prenatal stress alter sexual differentiation of the brain and reproductive behavior in male rat offspring. Psychoneuroendocrinology 38:1618-1629

80. Maccari S, Krugers HJ, Morley-Fletcher S, Szyf M, Brunton PJ (2014) The consequences of early-life adversity: neurobiological, behavioural and epigenetic adaptations. J Neuroendocrinol 26:707723

81. Lucassen PJ, Bosch OJ, Jousma E (2009) Prenatal stress reduces postnatal neurogenesis in rats selectively bred for high, but not low, anxiety: possible key role of placental 11 beta-hydroxysteroid dehydrogenase type 2. Eur J Neurosci 29:97-103

82. Laurent L, Deroy K, ST-Pierre J, Côte F, Sanderson JT, Vaillancourt C (2017) Human placenta expresses both peripheral and neuronal isoform of tryptophan hydroxylase. Biochimie 140: $159-165$

83. Muller CL, Anacker AM, Rogers TD (2017) Impact of maternal serotonin transporter genotype on placental serotonin, fetal forebrain serotonin, and neurodevelopment. Neuropsychopharmacology 42: $427-436$

84. Bonnin A, Levitt P (2011) Fetal, maternal, and placental sources of serotonin and new implications for developmental programming of the brain. Neuroscience 197:1-7

85. Rayen I, Steinvusch HW, Charlier TD, Pawluski JL (2014) Developmental fluoxetine exposure facilitates sexual behavior in female offspring. Psychopharmacology 231:123-133 
86. Pawluski JL, Rayen I, Niessen NA (2012) Developmental fluoxetine exposure differentially alters central and peripheral measures of the HPA system in adolescent male and female offspring. Neuroscience 220:131-141

87. Pawluski JL, Brain UM, Underhill CM, Hammond GL, Oberlander TF (2012) Prenatal SSRI exposure alters neonatal corticosteroid binding globulin, infant cortisol levels, and emerging HPA function. Psychoneuroendocrinology 37: 1019-1028
88. Bodnar LM, Wisner KL (2005) Nutrition and depression: implications for improving mental health among childbearing-aged women. Biol Psychiatry 58:679-685

89. Swain JE, Ho SS, Rosenblum KL, Morelen D, Dayton CJ, Muzik M (2017) Parent-child intervention decreases stress and increases maternal brain activity and connectivity during own baby-cry: an exploratory study. Dev Psychopathol 29:535-553

90. Angelotta C, Wisner KL (2017) Treating depression during pregnancy: are we asking the right questions? Birth Defects Res 109: 879-887 